\title{
MORADIA: UM HABITAT SAUDÁVEL PARA A PANDEMIA?
}

\author{
HOUSING: A HEALTHY HABITAT FOR PANDEMIC?
}

\author{
Maíra Longhinotti Felippe ${ }^{1}$, Raphaela Walger da Fonseca ${ }^{1}$, Fernanda Machado Dill \\ Angela Favaretto ${ }^{2}$, Vanessa Goulart Dorneles ${ }^{3}$, Amanda Silveira Correa ${ }^{3}$, Fernando \\ Oscar Ruttkay Pereira ${ }^{1}$
}

\section{RESUMO:}

A pandemia de COVID-19 impôs novos modos de relação entre as pessoas e seus ambientes de vida e nos desafiou a avaliar e repensar o habitat humano de modo a acomodar e dar suporte a tais mudanças. Assim, o objetivo deste trabalho é abordar características físicas do ambiente de moradia em sua relação com a satisfação ambiental no contexto da quarentena. Para tal, são apresentados os resultados de uma pesquisa da qual participaram 1858 pessoas, majoritariamente da região Sul do Brasil, realizada por meio de questionário online aplicado ao longo do período de abril a maio de 2020, num momento de maior isolamento social no país. Os participantes caracteristicamente avaliaram a própria moradia de forma positiva, porém aspectos ligados à oferta de espaço e de acesso a elementos naturais (como plantas e luz natural) estiveram entre os mais citados como características deficientes da habitação. Além disso, condições inadequadas de iluminação estiveram relacionadas a alterações de padrão de sono ou nível de alerta. Observou-se ainda que moradores de casas, bem como residentes de habitações com mais quartos, apresentaram avaliações mais positivas da moradia como lugar para se passar a quarentena. Essas relações foram discutidas a partir do conhecimento acerca do papel que o controle da privacidade e o acesso a elementos naturais têm para a promoção de saúde e bem-estar, e por conseguinte, da satisfação. O estudo põe em ênfase a responsabilidade da arquitetura na promoção da qualidade de vida, permitindo repensar para um futuro próximo a adequabilidade dos contextos ambientais às condições do habitar humano.

PALAVRAS-CHAVE: bem-estar; luz natural; privacidade; habitação; COVID-19.

\section{ABSTRACT:}

The COVID-19 pandemic imposed new relations between people and their living environments and challenged us to review and rethink the human habitat to accommodate and support such changes. The aim of this work is to address physical characteristics of the living environment in relation to environmental satisfaction in the context of quarantine. To this end, we present the results of a survey which was attended by 1858 people, mostly from South Brazil, conducted through an online questionnaire applied from April to May 2020, at a time of greater social isolation in the country. Participants typically evaluated their own housing in a positive way, but aspects linked to the provision of space and access to natural elements (such as plants and natural light) were among the most frequently cited as deficient characteristics of housing. In addition, inadequate lighting conditions were related to changes in sleep patterns or alertness. It was also observed that residents of houses, as well as residents of houses with more rooms, presented more positive ratings of the house as a place to spend the quarantine. These relations were discussed based on the knowledge about the role that the control of privacy and access to natural elements have for the promotion of health and well-being, and therefore, satisfaction. The study highlights the responsibility of architecture in promoting quality of life, allowing to rethink for the near future the adequacy of environmental contexts to the conditions of human inhabit.

KEYWORDS: well-being; daylight; privacy; housing; COVID-19.

How to cite this article:

FELIPPE, M. L.; FONSECA, R. W.; DILL, F. M.; FAVARETTO, A.; DORNELES, V. G.; CORREA, A. S.; PEREIRA, F. O. R. Moradia: um habitat saudável para a pandemia? Gestão \& Tecnologia de Projetos. São Carlos, v16, n4, 2021. https://doi.org/10.11606/gtp.vXiY.178708 


\section{INTRODUÇÃO}

0 mundo enfrenta um dos maiores desafios do século desde março de 2020 quando a Organização Mundial da Saúde (OMS) declarou a COVID-19 como pandemia (SCHMIDT et al., 2020). Frente à alta taxa de transmissão do vírus, os conhecimentos ainda limitados sobre padrões de transmissibilidade, infectabilidade, letalidade e mortalidade, além da diversidade de quadros de evolução clínica (LIMA et al., 2020), tem-se buscado diversas estratégias com o objetivo de frear a doença. 0 distanciamento social, o uso de máscaras, a utilização de álcool gel e outras medidas de higiene pessoal são algumas destas estratégias.

Em locais onde as taxas de transmissão continuam aumentando, em determinados períodos e como uma alternativa mais drástica contra a propagação do vírus, tem sido adotado o isolamento social, que prevê a permanência das pessoas em suas residências por longos períodos. 0 contexto de confinamento residencial e as incertezas sobre a doença apresentamse como fatores geradores de insegurança, estresse e mal-estar, afetando a saúde mental e física das pessoas. Por outro lado, entende-se que a boa qualidade das residências e a satisfação dos moradores com o espaço no qual estão confinados podem contribuir sobremaneira para o sentimento de bem-estar, impactando positivamente na saúde.

Antes da pandemia, as necessidades habitacionais cotidianas (espaço para trabalhar, contato com ambientes naturais, luz do Sol, etc.) podiam ser compartilhadas com espaços externos à residência, contudo, agora, surge a necessidade de certa autonomia nos espaços de morar. Assim, o objetivo deste trabalho é abordar características físicas do ambiente de moradia e sua relação com a satisfação para com o lugar no contexto da pandemia. Satisfação ambiental, consonante com a discussão de Moreno e Pol (1999, p. 32), é entendida aqui como o contentamento que decorre da compatibilidade entre o que se tem e o que se espera ou deseja, ou seja, do ajuste entre as características contextuais e o modo como as pessoas percebem suas expectativas e necessidades em relação ao ambiente.

Pretendeu-se investigar a satisfação com a residência vinculada à questão do bem-estar e da saúde, a partir dos conhecimentos acerca do importante papel que o controle sobre a privacidade e o contato com elementos naturais exteriores têm para promover e aumentar a qualidade de vida. Para isto, são apresentados os resultados de uma pesquisa realizada de forma online no período de abril a maio de 2020, num momento de maior reclusão social no Brasil.

\section{A MORADIA E O BEM-ESTAR NA PANDEMIA}

Originalmente, as habitações foram concebidas com a função de proteger seus habitantes de intempéries. No Dicionário da Arquitetura Brasileira, Corona e Lemos (1972, p. 257) definem habitação como "lugar no qual se habita. Constitui em arquitetura, o abrigo ou invólucro que protege o homem, favorecendo sua vida no duplo aspecto material e espiritual. Ato ou efeito de habitar. Morada. Residência."

Sendo então a proteção função primordial da habitação, se essa não oferece condições de salubridade a seus habitantes, ela não comporta o habitar e descaracteriza-se de sua etimologia. Enquanto habitação, as edificações podem ser abordadas como a terceira pele do homem, sendo a primeira, a epiderme; a segunda, a vestimenta que o protege do meio ambiente em que habita; e a terceira, a habitação propriamente dita (MIGUEL, 2002).

Em tempos de pandemia, essa condição do morar se tornou ainda mais evidente na rotina das pessoas, essa terceira pele passou a fazer mais sentido e estar ainda mais presente para todos. 0 fato é que as rotinas familiares, sociais e laborais foram alteradas com a pandemia. Os ambientes residenciais que por vezes eram direcionados em sua maioria para o descanso e o 
convívio familiar, agora estão sendo palco de todo tipo de atividade, incluindo uma infinidade de atividades virtuais. As pessoas passaram a apropriar-se ainda mais de suas residências, adaptando suas vidas e seus espaços a novas atividades, coabitando e compartilhando mais tempo e mais funções dentro da residência. Todos esses fatores podem ser considerados como geradores de estresse humano, especialmente em razão de uma maior dificuldade de controle sobre a privacidade. Quanto mais o tempo passa, mais consequências deste período de isolamento social, aglomeração residencial e falta de contato com o exterior e com a luz natural têm sido sentidas pelas pessoas.

Conforme explicam Cavalcante e Pinheiro (2018), a privacidade pode ser entendida como uma forma de controle de contato físico, mas também informacional (relativo à troca de informações), exercido por uma pessoa ou um grupo de pessoas. De acordo com Valera e Vidal (1998), isolamento, solitude, anonimato, reserva e intimidade são formas de manifestação da privacidade, em geral conquistadas com o auxílio de estratégias verbais e não verbais, bem como ambientais e relativas às normas sociais, que atuam para transformar a privacidade real (obtida) naquela ideal (esperada ou desejada). Um desequilíbrio nessa equação poderia ser responsável por sentimentos de apinhamento (menor privacidade do que se deseja) ou mesmo isolamento (maior privacidade do que se deseja), com consequências para o bem-estar da pessoa.

A territorialidade, entendida como um padrão de comportamento baseado na percepção de controle de um lugar, que pode implicar ações de ocupação, defesa, personalização e sinalização de um território, é considerada um mecanismo para a regulação da privacidade e as interações com outras pessoas (HIGUCHI; THEODOROVITZ, 2018; VALERA; VIDAL, 1998). De modo semelhante, o espaço pessoal, conceito criado por Robert Sommer $(1973 ; 2018)$ e caracterizado como espaço invisível que envolve um indivíduo, no qual não se pode adentrar sem causar desconforto, também se constitui como forma de controle de contato físico e informacional (TORVISCO, 1998).

Além dos aspectos ligados ao controle sobre a privacidade em família, outras circunstâncias podem ser consideradas como fatores produtores de estresse no contexto do distanciamento social. Um tempo maior de reclusão nas residências, por exemplo, muitas vezes implica na diminuição de contato com áreas verdes livres e elementos naturais em geral, tal qual a luz natural, especialmente para os que residem em habitações que não oferecem áreas externas de uso privativo. Sabe-se, no entanto, do papel que esses espaços possuem, não apenas para a redução do estresse, mas também para a produção de bem-estar de maneira global. A influência da natureza e dos elementos naturais sobre o bem-estar tem sido o foco de atenção de pesquisadores dedicados ao estudo dos chamados 'ambientes restauradores', ou seja, ambientes que permitem ou promovem o processo de restauração. Em outras palavras, o processo através do qual há restauração de recursos afetados pelas demandas diárias a que estamos submetidos (HARTIG, 2011).

Atualmente, no campo da Psicologia Ambiental, duas teorias têm recebido destaque por procurarem descrever as razões e os processos pelos quais os ambientes naturais consentem ou mesmo suscitam a restauração. A Teoria Psicoevolucionista ou Teoria da Recuperação do Estresse, de Roger Ulrich (Cf. ULRICH et al., 1991), demonstra que as pessoas estão biologicamente preparadas para reagir rápida, imediata e positivamente a cenários que tenham sido favoráveis ao desenvolvimento dos seres humanos enquanto espécie, a saber, os cenários naturais. Tal reação ambiental - traduzida por resposta afetiva que desmobiliza sistemas fisiológicos e psicológicos alterados pela condição de estresse - teria sido importante ao longo do processo evolutivo pelo fato de possibilitar que os esforços humanos fossem então destinados a práticas fundamentais para a manutenção da vida, como o descanso, o sono e a alimentação. 
Também Rachel e Stephen Kaplan, em sua Teoria da Restauração da Atenção (Cf. KAPLAN, 1995), defendem que os cenários naturais favorecem a recuperação do recurso da atenção direta e do processo inibitório de distrações, essenciais para o desenvolvimento do trabalho humano e, no entanto, sujeitos a fadiga. A fadiga da atenção direta está ligada a uma série de efeitos sobre o bem-estar, incluindo risco de acidentes, estados de irritação e estresse. Os autores explicam que a natureza permite a restauração atencional dirigida pelo fato de mobilizar um tipo diferente de atenção, uma atenção sem esforço, conhecida como involuntária ou de fascinação. Além disso, possui propriedades que potencializam esse processo, como a capacidade de promover um certo distanciamento psicológico em relação aos contextos diários demandantes (afastamento); favorecer oportunidades para a exploração ambiental, circunstância na qual se pode engajar a mente por longos períodos, de modo coerente e ordenado (extensão); e propiciar compatibilidade de uso frente aos interesses das pessoas (compatibilidade).

Assim, sob essas e outras perspectivas, um número crescente de estudos tem demonstrado empiricamente o papel da natureza na promoção do bem-estar, incluindo efeitos positivos sobre estados psicofisiológicos e ocorrência de doenças ligadas a estresse (GRAHN; STIGSDOTTER, 2003; TSUNETSUGU et al., 2013; ULRICH, 1981; ULRICH et al., 1991); humor e reflexão sobre metas pessoais (ROE; ASPINALL, 2011); número de reclamações sobre a saúde física e percepção de estado geral de saúde (VAN DEN BERG et al., 2010). Hartig e colegas (2003), por exemplo, descobriram que a restauração do estresse e da atenção dirigida foi promovida quando jovens adultos foram expostos ao ambiente natural. Nesse mesmo estudo, o ambiente urbano, em contrapartida, produziu efeitos psicofisiológicos negativos, corroborando outras investigações em que natureza e urbanidade foram confrontadas, como é o caso das pesquisas de Hauru et al. (2012), e Van den Berg et al. (2014), todas apontando para o importante papel restaurador dos cenários naturais. No contexto brasileiro, em um ambiente hospitalar pediátrico, Felippe e colegas (2020) encontraram que o acesso visual através das janelas ao ambiente exterior, em particular ao ambiente natural, esteve associado a todos os significados ambientais ligados ao processo de restauração afetiva do estresse. Também Silveira (2017) constatou que os elementos naturais e os espaços abertos foram os principais aspectos citados como favorecedores do bem-estar por trabalhadores de um hospital de custódia, sendo a natureza parte da identidade de ambiente restaurador desses profissionais.

A natureza, além de permitir ou promover a restauração a partir das condições de estresse e de fadiga da atenção, o que representa uma significativa colaboração para o bem-estar, constitui-se como área para lazer, circulação, atividade física, descanso e entretenimento, o que favorece o bem-estar físico e psíquico dos cidadãos; o amadurecimento emocional; as relações sociais saudáveis; um maior engajamento social e oportunidade de socialização (CASTONGUAY; JUTRAS, 2009; CHIESURA, 2004; HANSMANN; HUG; SEELAND, 2007; LEE; MAHESWARAN, 2011; SUGIYAMA et al., 2008). Também os benefícios ecossistêmicos de regulação proporcionados pela natureza, como a purificação do ar e das águas, a regulação do clima e dos ruídos, por exemplo, (HAINES-YOUNG; POTSCHIN, 2018) dão suporte ao bem-estar humano.

Ainda sobre a relação do bem-estar associado ao contato com a natureza, ressalta-se a importância do elemento luz. A exposição à luz natural é essencial à qualidade de vida, já que o ser humano se desenvolveu exposto à luz do dia. Além dos aspectos psicológicos, os efeitos da luz são fortemente relacionados à saúde biológica das pessoas, em especial pela sua influência no ritmo circadiano e no sistema endócrino (CIE, 2003, 2009; REPPERT; WEAVER, 2002). A exposição à luz é frequentemente reportada como terapêutica, sendo prescrita como um antidepressivo natural, a exemplo dos tratamentos para o transtorno afetivo sazonal (VIOLA, et al., 2008). Isso porque a produção de serotonina, um hormônio neurotransmissor 
associado ao bem-estar e a aspectos biopsíquicos, também influente no ritmo circadiano, depende da exposição a níveis elevados de luz (YOUNG, 2007).

A luz natural é a que possui composição e variação espectral mais adequada às funções biológicas humanas. Isso porque evoluímos adaptando-nos às condições de luminosidade resultante de longos períodos de exposição a essa fonte de luz, bem como aos ciclos naturais de claridade e escuridão. Tal padrão de exposição resultou em nosso ritmo circadiano. A alternância de luz e escuridão regula a produção de melatonina, responsável pelo ajuste desse ritmo, bem como da reprodução, sistema imunológico e pressão arterial (ALTUN; UGURALTUN, 2007). 0 ritmo circadiano regula diversas funções como sono e vigília, nível de alerta, humor, supressão e secreção de hormônios e a temperatura corporal central, entre outras (KONIS, 2019). Segundo Reppert e Weaver (2002), quando esse sistema não está em ordem, podem-se observar consequências genéticas e psicofisiológicas. Um ritmo circadiano desregulado é associado a distúrbios do sono, tais como insônia e sonolência excessiva, ao aumento do risco de diabetes e até mesmo a alguns tipos de câncer (STEVENS; REA, 2001).

Sob a ótica da arquitetura, a associação da luz do dia ao bem-estar é percebida por meio do aumento da produtividade e a melhora da saúde dos ocupantes das edificações iluminadas naturalmente. Pesquisas têm apresentado que espaços construídos que possuem associação de iluminação natural e com o contato com o espaço exterior, há melhora do desempenho de alunos em sala de aula (DEMIR; KONAN, 2013; HESCHONG, 2003a); e aumento da produtividade em estabelecimentos de escritório (HESCHONG, 2003b; LOFTNESS et al., 2008; MAYHOUB; CARTER, 2010). Existem evidências ainda de benefícios relacionados ao estado de saúde de pacientes em hospitais (BEAUCHEMIN; HAYS, 1996, 1998; CHOI; BELTRAN; KIM, 2012; WALCH et al., 2005). Por outro lado, edificações que não proporcionam iluminação natural adequada privam seus ocupantes da manutenção à sua saúde e do bem-estar enquanto nelas permanecem. Tal constatação foi agravada no cenário da pandemia, dada a restrição de mobilidade, o que dificultou a compensação das deficiências das residências a esse respeito. Pesquisas realizadas no Brasil, uma com funcionárias de lojas (MARTAU, 2009) e outra com funcionárias de um hospital (HARB; HIDALGO; MARTAU, 2015), identificaram a relação entre a possibilidade de exposição à luz natural oferecida pela edificação e impactos nos processos fisiológicos de suas ocupantes. Ambos os estudos indicaram relação entre a poluição luminosa e a falta de exposição à luz natural, associada aos ambientes de permanência das funcionárias, a níveis altos de cortisol e baixos de melatonina durante a noite, e estes, por sua vez, a problemas no sono e sintomas de depressão.

O contexto da pandemia surgiu num momento em que se via um crescimento da construção imobiliária com foco em espaços de convívio e espaços funcionais coletivos em edifícios residenciais e muitas vezes com redução da unidade habitacional. No entanto, agora tais unidades precisam absorver mais tempo de permanência e mais atividades. Estas reflexões sobre as mudanças trazidas pelo novo modo de morar levaram ao desenvolvimento da pesquisa que buscou avaliar a satisfação das pessoas em relação às suas moradias em diferentes aspectos.

\section{MÉTODO}

Esta pesquisa foi desenvolvida por meio de um levantamento de dados com abordagem quantitativa. Para tanto, foi aplicado um questionário online com o suporte da plataforma Survio, composto por 41 perguntas, caracterizado conforme Figura 1 e disponível para acesso e resposta entre 26 de abril a 26 de maio de 2020. A distribuição do questionário foi realizada através de e-mail, por whatsapp e redes sociais: Instagram e Facebook. Todas as pessoas que receberam a divulgação poderiam ou não concordar em responder ao questionário, pois em sua página de introdução havia uma explicação sobre o tempo estimado de resposta e o número 
de perguntas a serem respondidas. O questionário, bem como a descrição do projeto de pesquisa, também ficou disponível para conhecimento e divulgação através da plataforma wordpress.

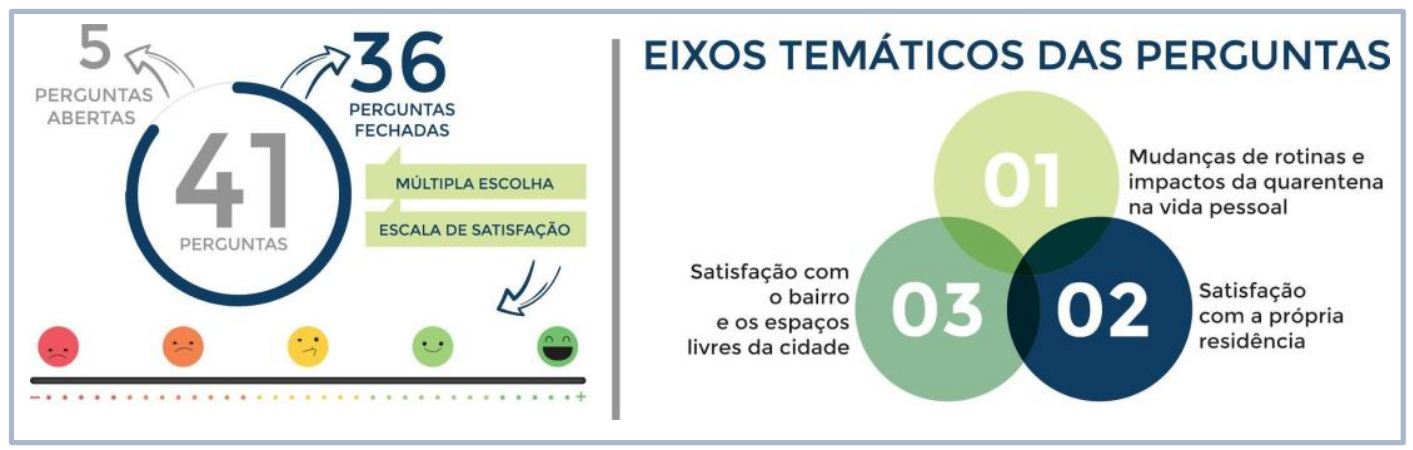

Das 41 perguntas, as primeiras quinze consistiam em uma caracterização da amostra de respondentes, incluindo alguns aspectos de identificação, forma de moradia, número de moradores na casa, entre outros. Destas, cinco perguntas abertas foram incluídas para identificar a idade, a cidade, o bairro e a profissão dos respondentes, sendo as demais perguntas fechadas.

Vale ressaltar que o foco deste artigo é o Eixo Temático 2 - Satisfação com a própria residência (conforme Figura 1), composto por questões que, ao longo do trabalho, serão relacionadas com a caracterização da amostra e aspectos do Eixo Temático 1, relativo às mudanças de rotinas durante a quarentena (e.g. alterações no padrão de sono).

Dessa forma, o estudo aqui relatado toma como base as seguintes perguntas destinadas à caracterização da amostra de respondentes: Qual a sua idade? Gênero? Você faz parte de algum grupo de risco para COVID-19? Sua profissão permite que trabalhe em casa? Em qual estado você mora? Qual o tipo de residência em que mora? Quantos quartos sua casa possui?

Também são consideradas as perguntas que seguem, sendo as três primeiras ligadas ao Eixo Temático 2 (satisfação com a residência), e a última ao Eixo Temático 1 (mudanças de rotina e impactos da quarentena): Como você avalia sua moradia no que diz respeito a um lugar para passar a quarentena? (escala de avaliação); Quais características da sua casa são desagradáveis? (seleção de múltiplas respostas); De que lugares/situações sente falta em casa? (seleção de múltiplas respostas); Você percebeu alterações no seu padrão de sono durante a quarentena? (seleção de uma resposta entre múltiplas opções). Todas as respostas relatadas neste trabalho foram dadas a partir de listas de múltiplas opções oferecidas ao respondente no momento de preenchimento do questionário.

Os dados obtidos foram examinados por meio de análise estatística descritiva e relacional, utilizando-se o software livre GNU PSPP (FREE SOFTWARE FOUNDATION, 2016). 0 projeto de pesquisa teve sua aprovação pelo Comitê de Ética em pesquisa (CAAE: 31344520.8.1001.5346) via Plataforma Brasil. Número do Parecer: 4.008.294.

\section{RESULTADOS E DISCUSSÃO}

Participaram do estudo 1858 pessoas, majoritariamente da região Sul do Brasil (93,5\%) e mulheres $(73,5 \%)$, não pertencentes ao grupo de risco para Covid-19, com média etária de 40 anos, como apresentado na Figura 2. Destaca-se ainda, que a maioria dos respondentes afirmou ter a possibilidade de trabalhar em casa no período da pandemia $(81,24 \%)$ e reside em bairros periféricos (81,2\%). Quanto ao tipo da residência, equilibram-se moradores de casa ou sítio
Figura 1. Caracterização do questionário.

Fonte: Elaboração própria, 2020. 
(44,03\%); e apartamentos, flats ou quitinetes (55,97\%). Dentre 1842 respondentes, 45,53\% moram em apartamento.

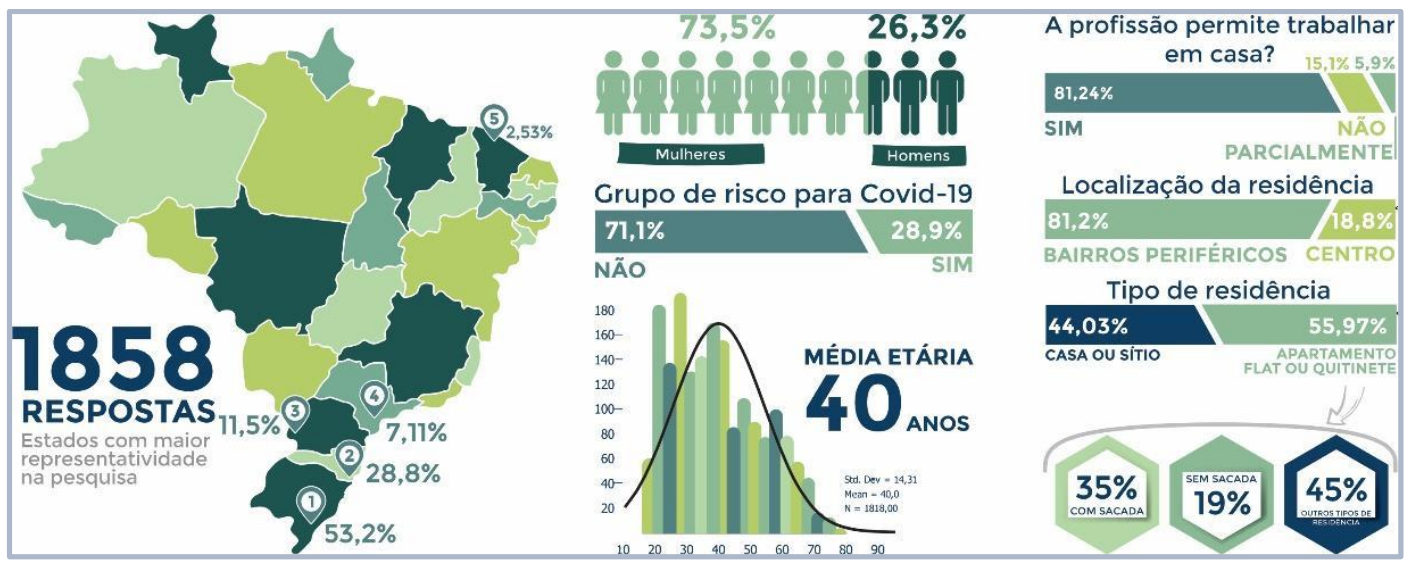

Como lugar para passar a quarentena, os participantes caracteristicamente avaliaram a própria moradia de forma positiva, tendo-se obtido média de votos igual a 8,12 (DP =1,95; $\mathrm{N}=1858)$, em uma escala de 1 a 10, em que 1 corresponde à pior avaliação e 10, à melhor. Considerando que um local de moradia com mais espaço disponível e que oferece maior possibilidade de acesso a áreas ao ar livre privativas pode auxiliar no controle de aspectos ligados à privacidade e a conflitos de uso, bem como proporcionar maiores oportunidades de restauração pelo contato com elementos naturais, como a luz natural e eventualmente áreas vegetadas, foi hipótese do presente estudo que residentes em casas, casas de condomínio, sítios ou áreas rurais, assim como residentes de habitações com mais quartos fariam uma avaliação mais positiva da residência como lugar para passar a quarentena do que pessoas residentes em apartamentos com ou sem sacada, flats e quitinetes, ou moradores de habitações com menos quartos.

Para testar essa hipótese foram formados primeiramente dois grupos independentes de respondentes: (a) o grupo de pessoas residentes em casa, casa de condomínio, sítio ou áreas rurais; (b) o grupo de pessoas residentes em apartamento com ou sem sacada, flat ou quitinete. As análises descritivas dos dados de avaliação da moradia para cada um dos dois grupos revelaram que a média dos escores de avaliação obtidos de quem mora em casa ou áreas rurais $(n=811)$ foi igual a 8,63 (DP = 1,79; $M d n=9,00 ; A=9,00)$, em uma escala de 1 a 10 , o que corresponde a uma avaliação positiva. Em contrapartida, entre os residentes de apartamentos, flats e quitinetes ( $n=1031$ ), esse valor foi equivalente a 7,71 (DP =1,98; $M d n=8,00 ; A=9,00)$, resultado que revela uma avaliação ainda positiva da residência, porém, inferior àquela realizada pelo outro grupo.

Para verificar se houve uma diferença entre grupos com relação à avaliação da residência como lugar para passar a quarentena, valeu-se do teste de Mann-Whitney (U). Assim, obteve-se U = $289309,00(\mathrm{z}=-11,68)$ com $p$ menor que 0,001. Esse resultado confirma a hipótese de que os dois grupos foram diferentes quanto à avaliação, tendo os residentes de casas, casas em condomínios, sítios e áreas rurais avaliado melhor a própria moradia. Como a diferença é estatisticamente significativa, pode-se considerar que a probabilidade desse resultado ter acontecido por erro amostral é pequena.

Em um segundo momento, na mesma direção, outros dois grupos foram formados: (a) o grupo de pessoas residentes em moradias com até dois quartos; e (b) o grupo de pessoas residentes em habitações com mais de dois quartos. O Teste de Mann-Whitney (U), para amostras independentes, também identificou uma relação entre a variável 'avaliação da moradia' e o tipo de residência, se com menos ou mais dormitórios. Nas habitações com mais de dois
Figura 2. Caracterização da amostra.

Fonte: Elaboração própria, 2020. 
dormitórios, a média dos escores de avaliação da residência como lugar para passar a quarentena foi 8,47 ( $D P=1,76 ; A=9,00 ; M d n=9,00 ; n=1152$ ), enquanto que, nas habitações com até dois quartos, a média foi inferior, igual a 7,54 (DP =2,10; $A=9,00 ; \mathrm{Mdn}=8,00 ; \mathrm{n}=$ 706). Obteve-se $\mathrm{U}=295466,00(\mathrm{z}=-10,19 \mathrm{com}$ um valor de $p$ menor que 0,001 , o que indica diferença entre as duas condições no que diz respeito à avaliação da residência. Moradores de habitações com mais de dois quartos avaliaram mais positivamente a moradia que aqueles de habitações com menos quartos.

Esses resultados são corroborados por outras questões investigadas nesta pesquisa. Considerando-se a amostra total de respondentes, quando perguntados sobre características desagradáveis da casa, mais pessoas informaram não haver qualquer aspecto desagradável, o que correspondeu a $22,21 \%(n=719)$ de um total de 3238 menções. No entanto, quando aspectos negativos da casa foram mencionados, estes estiveram mais ligados a pouco espaço de maneira geral $(13,09 \% ; n=424)$, ausência de horta caseira $(12,75 \% ; n=413)$, ausência de plantas decorativas $(7,66 \% ; n=248)$, pouca iluminação $(7,10 \% ; n=230)$ e falta de decoração $(6,86 \% ; n=222)$. Isto é, características ligadas à falta de espaço e de acesso a elementos naturais (como plantas e luz natural) estiveram entre as mais citadas ao se tratar de aspectos desagradáveis da habitação.

Tais achados também são suportados por aqueles obtidos a partir da questão "De que lugares/situações sente falta em casa?". Constatou-se alta frequência de respostas indicativas de que a casa é um lugar satisfatório (14,38\% de um total de 3775 citações), observando-se em contrapartida também referências à falta de contato com a natureza $(19,68 \%)$, de um lugar para tomar sol $(14,68 \%)$ ou realizar atividades físicas $(14,01 \%)$. A ausência de um local para trabalhar ou estudar de forma isolada $(11,47 \%)$ ou mesmo para ficar em silêncio/sozinho $(9,11 \%)$ também recebeu destaque, fato que sustenta a percepção de falta de espaço como aspecto problemático nas residências, tal qual já identificado anteriormente.

De acordo com as hipóteses, é possível que morar em casa, casa em condomínio fechado ou sítio acarrete mais frequentemente maiores oportunidades de contato com áreas externas e elementos naturais. Isso também daria aos moradores maior possibilidade de restauração e, por conseguinte, maior bem-estar e satisfação com a própria residência, como lugar que atende necessidades fisiológicas e psicológicas durante o período de confinamento. Como função adaptativa da espécie humana, tal como defendem Ulrich e colegas (1991), a restauração a partir de uma condição de estresse ou de fadiga configura-se como processo necessário à manutenção da saúde e da qualidade de vida, com efeitos sobre sensações de prazer, sentimento de contentamento, preferências e avaliações positivas em relação ao lugar que oportuniza tais relações e que, portanto, oferece melhores condições de vida. Condições que vão ao encontro, por exemplo, dos achados de Silveira (2017) em relação à produção de bemestar em um hospital de custódia e de Roe e Aspinall (2011), que encontraram mudanças positivas para o humor no espaço escolar, quando estudantes foram expostos ao ambiente de floresta.

Na mesma direção, morar em casa, casa em condomínio fechado ou sítio, bem como em habitações com maior número de quartos, pode possivelmente resultar em maior disponibilidade de espaço, o que facilitaria o exercício de controle sobre a própria privacidade, por meio do isolamento, da solitude (VALERA; VIDAL, 1998), do respeito ao território (HIGUCHI; THEODOROVITZ, 2018) e ao espaço pessoal (SOMMER, 1973; 2018), por exemplo. Tal controle parece ser especialmente importante no contexto de confinamento, quando membros da mesma família compartilham por mais tempo os espaços da moradia, enquanto desenvolvem afazeres e atividades dos mais variados tipos, entre trabalho, estudo, exercícios físicos, lazer e descanso, simultaneamente. A ausência da possibilidade de controle sobre a privacidade nesse contexto pode acarretar estresse e, em consequência, prejuízo à saúde e ao bem-estar, tal qual discutem Valera e Vidal (1998) e Higuchi e Theodorovitz (2018), com 
reflexos sobre as avaliações e julgamentos do indivíduo em relação ao próprio local de habitação.

Dando seguimento às análises previstas neste estudo, foram também investigadas questões relacionadas às condições de iluminação proporcionadas pelas residências, como acesso ao elemento natural 'luz', à saúde e ao bem-estar. No contexto desta pesquisa, a saúde e o bemestar foram abordados a lume do potencial da edificação para a manutenção do ritmo circadiano dos seus habitantes e consequentemente do funcionamento saudável dos sistemas corpóreos regulados por ele, bem como dos aspectos biopsíquicos associados à regulação desses sistemas. Foram observadas a percepção do morador em relação à oferta de luz no ambiente interno, seja por fontes naturais ou artificiais, e a possibilidade de contato com o exterior. Tal viés deu-se por muitos dos transtornos de sono relacionados à disfunção do ritmo circadiano (CRDS) serem causados pela falta de exposição suficiente a agentes sincronizadores externos, em especial, a luz natural; e pelo padrão inadequado de exposição à luz (MARTINEZ; LENZ; MENNA-BARRETO, 2008).

Dessa forma, foram avaliadas as relações entre características luminosas oferecidas pela habitação e alterações no padrão de sono e nível de alerta de seus ocupantes durante a quarentena. Testou-se a hipótese de que os entrevistados que elencaram a falta de iluminação entre as características desagradáveis de suas casas, teriam sofrido maior impacto associado a CRDS, nesse período. Entre os respondentes, 167 listaram a falta de iluminação entre as características desagradáveis de sua moradia $(\mathrm{N}=1363$, considerando-se apenas os participantes que responderam à questão). Destes que relataram falta de iluminação, $80,84 \%$ reportaram algum tipo de alteração do padrão de sono ou do estado de alerta. Para os respondentes neutros quanto à falta de iluminação, este percentual foi de 71,07\% (ver Tabela 1).

\begin{tabular}{|c|c|c|c|c|c|c|}
\hline \multirow[b]{3}{*}{$\begin{array}{r}\text { Característica desagradável da } \\
\text { residência }\end{array}$} & \multicolumn{6}{|c|}{ Alteração no padrão de sono ou estado de alerta } \\
\hline & \multicolumn{2}{|c|}{ Não } & \multicolumn{2}{|c|}{ Sim } & \multicolumn{2}{|c|}{ Total } \\
\hline & $\mathrm{N}$ & $\%$ & $\mathrm{n}$ & $\%$ & $\mathrm{n}$ & $\%$ \\
\hline Neutro & 346 & 28,93 & 850 & 71,07 & 1196 & 100 \\
\hline \multirow[t]{2}{*}{ Falta de iluminação } & 32 & 19,16 & 135 & 80,84 & 167 & 100 \\
\hline & 378 & 27,73 & 985 & 72,27 & 1363 & 100 \\
\hline
\end{tabular}

0 teste $\chi^{2}$ revelou haver evidências de uma associação entre falta de luminosidade e alteração no padrão de sono, ou no estado de alerta, durante a quarentena $\left(\chi^{2}=6,98 ; g l=1 ; p=0,008\right)$. Entretanto, o Coeficiente de Contingência de Pearson (C) obtido foi de 0,07, indicando um efeito pequeno de uma variável em relação à outra. Provavelmente, o efeito pequeno se deu pela interferência de outros fatores, como a própria condição estressora da pandemia, que por si só pode resultar em alteração do sono, inclusive nas habitações com iluminação adequada. 0 cálculo do risco relativo indicou que as pessoas que reportaram descontentamento por falta de iluminação têm 1,7 vezes mais chance de ter alteração no padrão de sono ou no estado de alerta do que os respondentes neutros, o que corrobora o importante papel da residência oferecer iluminação adequada à manutenção da saúde e à promoção de bem-estar de seus
Tabela 1. Associação entre a falta de iluminação e a alteração no padrão de sono ou estado de alerta.

Fonte: Elaboração própria, 2020. 
ocupantes. Tais resultados são convergentes com os resultados obtidos nas pesquisas de Martau (2009) e Harb, Hidalgo e Martau (2015) que relacionaram o padrão de exposição à luz a impactos na fisiologia humana. É importante salientar que a privação de sono ou o sono irregular a longo prazo podem causar prejuízo funcional, aumentar o risco de acidentes, levar à depressão e a transtornos de humor, resultando em sofrimento importante (MARTINEZ; LENZ; MENNA-BARRETO, 2008).

A regulação do ritmo circadiano humano depende da intensidade da iluminação, mas também do espectro luminoso associado ao horário e à duração da exposição à luz, entre outros (ANDERSEN; MARDAJEVIC E LOCKLEY, 2012; KONIS, 2017; LOCKLEY; BRAINARD E CZEISLER, 2003). Diante das evidências de que a iluminação natural é a mais apropriada para tal regulação, visto que o nosso sistema biológico evoluiu exposto à variação de suas tonalidades, e por conseguinte, responde melhor ao seu espectro, inferiu-se que pessoas que residissem em imóveis com maior possibilidade de contato com o exterior, teriam sofrido menor impacto associado a CRDS nesse período. Para averiguar se o tipo de habitação poderia estar relacionado a alterações no padrão de sono ou no nível de alerta, outras análises foram realizadas. Entre os respondentes, $27,66 \%$ declararam não ter percebido alteração no padrão de sono ou no estado de alerta, enquanto 72,34\% reportaram algum tipo de alteração ( $\mathrm{N}=$ 1352, considerando-se apenas os participantes que responderam à questão). Dentre os participantes que não perceberam nenhuma alteração, 40,91\% moram em casa ou sítio e $59,09 \%$ em apartamento, flat ou quitinete. Os resultados constam na Tabela 2.

\begin{tabular}{|c|c|c|c|c|c|c|}
\hline \multirow[b]{3}{*}{ Tipo de residência } & \multicolumn{6}{|c|}{ Alteração no padrão de sono ou estado de alerta } \\
\hline & \multicolumn{2}{|c|}{ Não } & \multicolumn{2}{|c|}{$\operatorname{Sim}$} & \multicolumn{2}{|c|}{ Total } \\
\hline & $\mathrm{n}$ & $\%$ & $\mathrm{n}$ & $\%$ & $\mathrm{n}$ & $\%$ \\
\hline Casa, sítio & 153 & 25,54 & 446 & 74,46 & 559 & 100 \\
\hline Apartamento, flat, quitinete & 221 & 29,35 & 532 & 70,65 & 753 & 100 \\
\hline Total & 374 & 27,66 & 978 & 72,34 & 1352 & 100 \\
\hline
\end{tabular}

O percentual de ocorrência de alteração do padrão de sono ou do estado de alerta foi superior tanto para aqueles que moravam em casa e sítio $(74,46 \%)$ quanto para aqueles que moravam em apartamento, flat ou quitinete $(70,65 \%)$. 0 teste $\chi^{2}$ revelou não haver evidências de uma associação entre o tipo de residência e alteração no padrão de sono ou no estado de alerta durante a quarentena $\left(\chi^{2}=2,42 ; \mathrm{gl}=1 ; \mathrm{p}=0,120\right)$.

A inexistência de relação indicada pelo teste estatístico pode justificar-se pela sobreposição de outros fatores. A priori, supõe-se o fator estresse, característico da própria pandemia, haja vista a ocorrência de alteração reportada por ambos os grupos. A posteriori, listam-se características arquitetônicas da residência incluindo a quantidade de aberturas, o tipo de acabamento das superfícies internas, a qualidade da iluminação, as características do entorno; e aspectos que independem da habitação, tais como os comportamentais (exposição à luz e aos ambientes externos), os psicológicos e os fisiológicos. Acrescenta-se que independentemente
Tabela 2. Associação entre 0 tipo de residência e a alteração no padrão de sono ou estado de alerta.

Fonte: Elaboração própria, 2020. 
do tipo de moradia, a alteração de rotina, de maneira geral, fez com que as pessoas permanecessem por mais tempo no interior das edificações, reduzindo de forma global o seu tempo de exposição à luz do dia.

Das análises realizadas pode-se também inferir que tanto casas como apartamentos podem oferecer condições internas adequadas ou inadequadas de iluminação natural em razão ao projeto de arquitetura e, nesse quesito, mais importante do que o tipo de moradia, é a sua qualidade no que tange à iluminação proporcionada. Tal afirmação, alinha-se com o que foi preconizado por Lockley, Brainard e Czeisler (2003) que demonstraram o impacto de fontes de luz, com diferentes distribuições espectrais, no ritmo circadiano humano. Dos resultados obtidos por estes autores, observa-se que o tipo de iluminação da residência (natural e artificial), associado ao horário, tempo, padrão e histórico de exposição à luz podem interferir sobremaneira nos processo fisiológicos de seus ocupantes.

\section{CONSIDERAÇÕES FINAIS}

Este trabalho teve o objetivo de abordar características físicas do ambiente de moradia - em particular, ligadas à oportunidade de controle da privacidade e contato com elementos naturais - e sua relação com a satisfação para com o lugar. Entende-se que a satisfação ambiental é um componente da qualidade de vida e resulta da experiência pessoal de habitar lugares promotores de bem-estar e saúde.

Como resultados desta investigação, obteve-se que os participantes caracteristicamente avaliaram a própria moradia de forma positiva, porém aspectos ligados à oferta de espaço e de acesso a elementos naturais (como plantas e luz natural) estiveram entre os mais citados enquanto características deficientes da habitação. Observou-se que moradores de casas, casas em condomínios e sítios, bem como residentes de habitações com mais quartos apresentaram avaliações mais positivas da moradia como lugar para se passar a quarentena que moradores de apartamentos, flats ou quitinetes, e residentes de habitações com menor número de quartos. Também se verificou que a percepção de condições inadequadas de iluminação no interior das edificações está relacionada com alterações de padrão de sono ou no nível de alerta. Essas relações foram discutidas a partir do conhecimento acerca do papel que o controle da privacidade e o acesso a elementos naturais têm para a promoção de saúde e bem-estar, e por conseguinte, da satisfação.

Neste trabalho, supôs-se que casas poderiam oferecer maiores possibilidades de acesso ao exterior e a elementos naturais (como a luz do Sol) que apartamentos, do mesmo modo que melhores condições de oferta de espaço. Claramente, essa suposição apresenta limitações, já que acesso ao exterior e amplitude são características que podem ser encontradas em ambos os tipos de residência. Sugerem-se, portanto, investigações futuras acerca das relações diretas que tais aspectos estabelecem com a satisfação. Outra limitação do estudo diz respeito ao fato de que a amostra investigada caracteristicamente acabou por não representar pessoas que moram em situação de vulnerabilidade. Desse modo, são necessárias pesquisas que levem essa condição em consideração.

Este estudo procurou lançar luz sobre alguns aspectos que podem ser relevantes na construção de um habitat saudável de forma geral e, especialmente, no contexto do isolamento social ocasionado pela pandemia de COVID-19, onde se verificou extenso período de permanência das pessoas dentro das edificações. Espaços apropriados a um controle adequado da privacidade, acesso ao exterior e a elementos naturais, bem como à quantidade e a qualidade de luz necessária à manutenção do funcionamento biológico do corpo humano, podem estar entre esses aspectos. Conhecê-los passa a ser indispensável ao desenvolvimento de novas habitações e à renovação das existentes, permitindo repensar para um futuro próximo a 
adequabilidade dos contextos ambientais às condições do habitar. A qualidade da arquitetura no que se refere aos aspectos sensoriais e perceptuais das pessoas pode determinar o quanto a moradia efetivamente as protegerá ou acabará por prejudicá-las. Essas considerações põem em ênfase a responsabilidade da arquitetura na promoção da saúde, bem-estar, satisfação e qualidade de vida dos cidadãos, evidenciando a dependência e a interrelação destes para com o ambiente.

\section{Agradecimentos}

Os autores Raphaela Walger da Fonseca (bolsa 151162/2019-0) e Fernando Oscar Ruttkay Pereira (bolsa 309394/2020-1) agradecem ao CNPq.

\section{Referências Bibliográficas}

ANDERSEN, M.; MARDALIEVIC, J.; LOCKLEY, S.W. A framework for predicting the nonvisual effects of daylight - Part I: photobiology-based model. Lighting Research and Technology, v. 44, n. 1, p. $37-$ 53, 2012. Disponível em: https://journals.sagepub.com/doi/10.1177/1477153511435961. Acesso em: 27 fev. 2021.

ALTUN, A.; UGUR-ALTUN, B. Melatonin: therapeutic and clinical utilization. International Journal of Clinical Practice, v. 61, n. 5, p. 835-45, 2007. Disponível em: https://pubmed.ncbi.nlm.nih.gov/17298593/. Acesso em: 27 fev. 2021.

BEAUCHEMIN, K. M.; HAYS, P. Sunny hospital rooms expedite recovery from severe and refractory depressions. Journal of Affective Disorders, v. 40, n. 1-2, p. 49-51, 1996. Disponível em: https://pubmed.ncbi.nlm.nih.gov/8882914/. Acesso em: $27 \mathrm{fev} .2021$.

BEAUCHEMIN, K.; HAYS P. Dying in the dark: sunshine, gender and outcomes in myocardial infarction. Journal of the Royal Society of Medicine, v. 91, n. 7, p 352-354, 1998. Disponível em: https://pubmed.ncbi.nlm.nih.gov/9771492/. Acesso em: 27 fev. 2021.

CASTONGUAY, G.; JUTRAS, S. Children's appreciation of outdoor places in a poor neighborhood. Journal of Environmental Psychology, v. 29, n. 1, p. 101-109, 1 mar. 2009. Disponível em: https://www.sciencedirect.com/science/article/abs/pii/S0272494408000418. Acesso em: $27 \mathrm{fev}$. 2021.

CAVALCANTE, S.; PINHEIRO, N. P. Privacidade. In: CAVALCANTE, S.; ELALI, G. A. (Orgs.). Psicologia ambiental: conceitos para a leitura da relação pessoa-ambiente. Petrópolis, RJ: Vozes, 2018. p. 197203.

CHIESURA, A. The role of urban parks for the sustainable city. Landscape and Urban Planning, $v$. 68, n. $1, \quad$ p. 129-138, 15 maio 2004. Disponível em: https://www.witpress.com/Secure/elibrary/papers/SC04/SC04034FU.pdf. Acesso em: 27 fev. 2021.

CHOI, J.-H.; BELTRAN, L. O.; KIM, H.-S. Impacts of indoor daylight environments on patient average length of stay (ALOS) in a healthcare facility. Building and Environment, v. 50, p. 65-75, 2012. Disponível em: https://www.sciencedirect.com/science/article/abs/pii/\$0360132311003593. Acesso em: 27 fev. 2021.

CIE. TC 6-11. Draft "Ocular lighting effects on human physiology, mood and behaviour". Commission Internationale d'Eclairage. 2003.2 Disponível em: http://www.elisirlin.com.ar/02 report\%20CIE\%20ocular\%20lighting\%20effects\%20611.pdf.

Acesso em: 27 fev. 2021. 
CIE. TC 6-11. Ocular lighting effects on human physiology, mood, and behavior (CIE 158:2004) ERRATUM (2009-Jan-21). Commission Internationale d'Eclairage. 2009.

CORONA, E.; LEMOS, C. A. C. Dicionário da arquitetura brasileira. São Paulo: EDART - São Paulo Livraria Editora Ltda., 1972.

DEMIR, A.; KONAN, N. Impact of daylighting on student and teacher performance. Journal of Educational and Instructional Studies in the World, v. 3, n. 1, fev./mar./abr. 2013. Disponível em: https://arastirmax.com/tr/system/files/dergiler/116392/makaleler/3/1/arastirmax-impactdaylighting-student-and-teacher-performance.pdf. Acesso em: 27 fev. 2021.

FELIPPE, M; HODECKER, M.; PICHETTI, D. Z. C. M; KUHNEN, A. Ambiente físico e significado ambiental no processo de restauração do estresse em quartos de internação pediátrica. Revista Projetar: Projeto e Percepção do Ambiente, v. 5, n. 1, p. 33-48, 2020. Disponível em: https://periodicos.ufrn.br/revprojetar/article/view/18767/12425. Acesso em: 27 fev. 2021.

FREE SOFTWARE FOUNDATION. GNU PSPP Statistical Analysis Software Release. Versão 1.2.0gOfb4db. [S.I.]: GNU, 2016.

GRAHN, P.; STIGSDOTTER, U. A. Landscape planning and stress. Urban Forestry \& Urban Greening, v. 2 , n. 1 , p. 1-18, jan. 2003. Disponível em: https://www.sciencedirect.com/science/article/abs/pii/S1618866704700199. Acesso em: 27 fev. 2021.

HAINES-YOUNG, R.; POTSCHIN, M. B. Common International Classification of Ecosystem Services (CICES) V5.1 and Guidance on the Application of the Revised Structure, 2018. Disponivel em: https://cices.eu/content/uploads/sites/8/2018/01/Guidance-V51-01012018.pdf. Acesso em: 26 nov. 2020.

HANSMANN, R.; HUG, S-M.; SEELAND, K. Restoration and stress relief through physical activities in forests and parks. Urban Forestry \& Urban Greening, v. 6, n. 4, p. 213-225, 15 nov. 2007. Disponível em: https://www.sciencedirect.com/science/article/abs/pii/S1618866707000623. Acesso em: 27 fev. 2021.

HARTIG, T.; EVANS, G. W.; JAMNER, L. D.; DAVIS, D. S.; GÄRLINGE, T. Tracking restoration in natural and urban field settings. Journal of Environmental Psychology, v. 23, n. 2, p. 109-123, 1 jun. 2003. Disponível em: https://www.sciencedirect.com/science/article/abs/pii/S0272494402001093. Acesso em: 27 fev. 2021.

HARTIG, T. Issues in restorative environment research: matters of measurement. In: FERNÁNDEZRAMÍREZ, B. et al. (Orgs.). Psicología ambiental 2011: entre los estudios urbanos y el análisis de la sostenibilidad. Almería: Universidad de Almería, p. 41-66, 2011.

HAURU, K.; LEHVÄVIRTA, S; KORPELA, K.; KOTZEA, D. J. Closure of view to the urban matrix has positive effects on perceived restorativeness in urban forests in Helsinki, Finland. Landscape and Urban Planning, v. 107, n. 4, p. 361-369, 30 set. 2012. Disponível em: https://www.sciencedirect.com/science/article/abs/pii/S0169204612002137. Acesso em: 27 fev. 2021.

HESCHONG, L. Windows and classrooms: a study of student performance and the indoor environment. Heschong Mahone Group - Public Interest Energy Research - California Energy Commission. Fair Oaks: outubro de 2003, p.111, 2003a. (P500-03-082-A-7).

HESCHONG, L. Window and offices: a study of office worker performance and the indoor environment. Heschong Mahone Group - Public Interest Energy Research - California Energy Commission. Fair Oaks: outubro de 2003, p.143, 2003b. (P500-03-082-A-9). 
HARB, F.; HIDALGO, M. P.; MARTAU, B. Lack of exposure to natural light in the workspace is associated with physiological, sleep and depressive symptoms. Chronobiology International, v. 32, n.3, p. 368-375, 2015. Disponível em: https://pubmed.ncbi.nlm.nih.gov/25424517/. Acesso em: 27 fev. 2021.

HIGUCHI, M. I. G.; THEODOROVITZ, I. J. Territorialidade(s). In: CAVALCANTE, S.; ELALI, G. A. (Orgs.). Psicologia ambiental: conceitos para a leitura da relação pessoa-ambiente. Petrópolis, RJ: Vozes, 2018. p. 228-236.

KAPLAN, S. The restorative benefits of nature: toward an integrative framework. Journal of Environmental Psychology, v. 15, n. 3, p. 169-182, set. 1995. Disponível em: https://www.sciencedirect.com/science/article/abs/pii/0272494495900012. Acesso em: 27 fev. 2021.

KONIS, K. A novel circadian daylight metric for building design and evaluation. Building and Environment. v. 113, p. 22-38, 2017. Disponível em: https://www.sciencedirect.com/science/article/abs/pii/S0360132316304498. Acesso em: 27 fev. 2021.

KONIS, K. A circadian design assist tool to evaluate daylight access in buildings for human biological lighting needs. Solar Energy. v. 191, p. 449-458, 2019. Disponível em: https://www.sciencedirect.com/science/article/abs/pii/S0038092X19308953. Acesso em: $27 \mathrm{fev}$. 2021.

LEE, A. C. K.; MAHESWARAN, R. The health benefits of urban green spaces: a review of the evidence. Journal of Public Health (Oxford, England), v. 33, n. 2, p. 212-222, jun. 2011. Disponível em: https://pubmed.ncbi.nlm.nih.gov/20833671/. Acesso em: 27 fev. 2021.

LIMA, D. S., et al. Recommendations for emergency surgery during the COVID - 19 pandemic. CJMB, v. $8, \quad$ n. 1 , p. 1-3, 2020. Disponível em: https://periodicos.unichristus.edu.br/jhbs/article/view/3176. Acesso em: 27 fev. 2021.

LOCKLEY, S. W.; BRAINARD, G. C.; CZEISLER, C. A. High sensitivity of the human circadian melatonin rhythm to resetting by short wavelength light. The Journal of Clinical Endocrinogy \& Metabolism. v. 88, n. 9, p. 4502-4505, 2003. Disponível em: https://pubmed.ncbi.nlm.nih.gov/12970330/. Acesso em: 27 fev. 2021.

LOFTNESS, V., et al. Building Investment Decision Support (BIDS ${ }^{\mathrm{Tm}}$ ): cost-benefit tool to promote high performance components, flexible infrastructures and systems integration for sustainable commercial buildings and productive organizations. Carnegie Mellon University Center for Building Performance and Diagnostics. Pittsburgh, p.29, 2008. Disponível em: https://www.semanticscholar.org/paper/BUILDING-INVESTMENT-DECISION-SUPPORT(BIDS\%E2\%84\%A2)-Tool-Faia-Hartkopf/6fac5fd459e56caa3bf6188d47cb5638f1e684fd. Acesso em: 27 fev. 2021.

MARTAU, B. T. A luz além da visão: iluminação e sua relação com a saúde e bem-estar de funcionárias de lojas de rua e de shopping centers em Porto Alegre. Tese. Pós-graduação da Faculdade de Engenharia Civil, Arquitetura e Urbanismo da Universidade Estadual de Campinas. Campinas, 2009. $504 \mathrm{p}$.

MARTINEZ, D.; LENZ, M. C. S; MENNA-BARRETO, L. Diagnóstico dos transtornos do sono relacionados ao ritmo circadiano. J. Bras. Pneumol. v. 34, n. 3, p. 173-180, mar. 2008. São Paulo. Disponivel em: $\quad$ https://www.scielo.br/scielo.php?script=sci arttext\&pid=S180637132008000300008. Acesso em: 27 fev. 2021. 
MAYHOUB, M. S.; CARTER, D. J. Towards hybrid lighting systems: a review. Lighting Research \& Technology, v. 42 , n. 1, p. 51-71, mar. 2010. Disponível em: https://journals.sagepub.com/doi/abs/10.1177/1477153509103724?journalCode=Irtd. Acesso em: 27 fev. 2021.

MIGUEL, C. M. J. Casa e lar: a essência da arquitetura. Vitrúvius. Arquitextos. 029.11. ano 03, out. 2002. Disponível em: https://www.vitruvius.com.br/revistas/read/arquitextos/03.029/746. Acesso em: 10 out. 2020.

MORENO, E.; POL, E. Nociones psicosociales para la intervención y la gestión ambiental. Barcelona: Universitat de Barcelona, 1999. Disponível em: http://www.publicacions.ub.edu/ficha.aspx?cod=08565. Acesso em: 13 out. 2020.

REPPERT, S. M.; WEAVER, D. R. Coordination of circadian timing in mammals. Nature, v. $418, \mathrm{n}$. 6901, p. 935-941, 2002.2 Disponivel em: https://www.nature.com/articles/nature00965\#: :text=Circadian\%20timing\%20in\%20mammals\% 20is,SCN\%20are\%20'clock\%20cells'. Acesso em: 27 fev. 2021.

ROE, J.; ASPINALL, P. The restorative outcomes of forest school and conventional school in young people with good and poor behaviour. Urban Forestry \& Urban Greening, v. 10, n. 3, p. 205-212, 1 jan. 2011. Disponível em: https://www.sciencedirect.com/science/article/abs/pii/S1618866711000318. Acesso em: 27 fev. 2021.

SILVEIRA, B. B. Estresse e restauração: Aspectos físicos e psicológicos de um hospital de custódia. Dissertação (Mestrado em Psicologia) - Departamento de Psicologia, Universidade Federal de Santa Catarina. Florianópolis, p. 146, 2017.

SOMMER, R. Espaço pessoal: as bases comportamentais de projetos e planejamentos. São Paulo: EPU, Editora da Universidade de São Paulo, 1973.

SOMMER, R. Espaço pessoal. In: CAVALCANTE, S.; ELALI, G. A. (Orgs.). Psicologia ambiental: conceitos para a leitura da relação pessoa-ambiente. Petrópolis, RJ: Vozes, p. 119-126, 2018.

SUGIYAMA, T.; LESLIE, E.; Giles-Corti, B.; Owen, N. Associations of neighbourhood greenness with physical and mental health: do walking, social coherence and local social interaction explain the relationships? Journal of Epidemiology \& Community Health, v. 62, n. 5, p. e9-e9, 1 maio 2008. Disponível em: https://jech.bmi.com/content/62/5/e9. Acesso em: 27 fev. 2021.

SCHMIDT, B.; CREPALDI, M. A.; BOLZE, S. D. A.; NEIVA-SILVA, L.; DEMENECH, L. M. Saúde mental e intervenções psicológicas diante da pandemia do novo coronavírus (COVID-19). Estud. psicol. (Campinas), Campinas, v. 37, e200063, 2020. Disponível em: https://www.scielo.br/scielo.php?script=sci arttext\&pid=S0103-166X2020000100501. Acesso em: 27 fev. 2021.

STEVENS R.; REA M. Light in the built environment: potential role of circadian disruption in endocrine disruption and breast cancer. Cancer Causes Control. v. 12, n. 3, p 279-287, 2001. Disponível em: https://pubmed.ncbi.nlm.nih.gov/11405333/. Acesso em: 27 fev. 2021.

TORVISCO, J. M. Espacio personal y ecología del pequeño grupo. In: ARAGONÉS, J. I. Y.; AMÉRIGO, M. (Orgs.). Psicología ambiental. Madrid: Pirâmide, p. 101-121, 1998.

TSUNETSUGU, Y.; LEE, J.; PARK, B-J; TYRVÄINEN, L.; KAGAWA, T.; MIYAZAKI, Y. Physiological and psychological effects of viewing urban forest landscapes assessed by multiple measurements. Landscape and Urban Planning, v. 113, p. 90-93, 1 maio 2013. Disponível em: 
https://www.sciencedirect.com/science/article/abs/pii/S0169204613000212. Acesso em: 27 fev. 2021.

ULRICH, R. S.; SIMONS, R. F.; LOSITO, B. D.; FIORITO, E.; MILES, M. A.; ZELSON, M. Stress recovery during exposure to natural and urban environments. Journal of Environmental Psychology, v. 11, n. 3, p. 201-230, set. $1991 . \quad$ Disponível em: https://www.sciencedirect.com/science/article/abs/pii/S0272494405801847. Acesso em: 27 fev. 2021.

ULRICH, R. S. Natural versus urban scenes: some psychophysiological effects. Environment and Behavior, v. 13, n. 5, p. 523-556, 1 set. 1981. Disponível em: https://journals.sagepub.com/doi/10.1177/0013916581135001. Acesso em: 27 fev. 2021.

VALERA, S.; VIDAL, T. Privacidad y territorialidad. In: ARAGONÉS, J. I. Y.; AMÉRIGO, M. (Orgs.). Psicología ambiental. Madrid: Pirâmide, p. 123-148, 1998.

VAN DEN BERG, A. E; JORGENSEN, A.; WILSON, E. R. Evaluating restoration in urban green spaces: Does setting type make a difference? Landscape and Urban Planning, v. 127, p. 173-181, 1 jul. 2014.

Disponível em: https://www.sciencedirect.com/science/article/abs/pii/S0169204614000966. Acesso em: 27 fev. 2021.

VAN DEN BERG, A. E.; MAAS, J.; VERHEIJ, R. A.; GROENEWEGEN, P. P. Green space as a buffer between stressful life events and health. Social Science \& Medicine, v. 70, n. 8, p. 1203-1210, 1 abr. $2010 . \quad$ Disponível em: https://www.sciencedirect.com/science/article/abs/pii/S0169204614000966. Acesso em: 27 fev. 2021.

WALCH, J. M.; RABIN, B. S.; DAY, R.; WILLIAMS, J. N.; CHOI, K.; KANG, J. D. The effect of sunlight on postoperative analgesic medication use: A prospective study of patients undergoing spinal surgery. Psychosomatic Medicine, v. 67, n. 1, p. 156-163, jan. 2005. Disponível em: https://pubmed.ncbi.nlm.nih.gov/15673638/. Acesso em: 27 fev. 2021.

YOUNG, S. N. How to increase serotonin in the human brain without drugs. Journal of Psychiatry \& Neuroscience, v. 32, n. 6, p. 394-399, 2007. Disponível em: https://www.ncbi.nlm.nih.gov/pmc/articles/PMC2077351/. Acesso em: 27 fev. 2021.
Maíra Longhinotti Felippe m.l.felippe@ufsc.br

Raphaela Walger da Fonseca raphawf@gmail.com

Fernanda Machado Dill fernanda.dill@gmail.com

Angela Favaretto arafavaretto@gmail.com

Vanessa Goulart Dorneles arq.vanessadorneles@gmail.co m

Amanda Silveira Correa amandaslvrcorrea@gmail.com

Fernando Oscar Ruttkay Pereira feco@arq.ufsc.br 